\title{
Harnessing Shape Effects for Adsorbate Signal Enhancement in Vibrational EELS
}

\author{
David Kordahl ${ }^{1}$ and Christian Dwyer ${ }^{1}$ \\ 1. Department of Physics, Arizona State University, Tempe, AZ 85287-1504, USA.
}

Raman spectroscopists have known for decades that rough surfaces, in the case of surface-enhanced Raman spectroscopy (SERS), or sharp metal tips brought near samples, in the case of tip-enhanced Raman spectroscopy (TERS), can cause enormous enhancements for vibrational signals of samples adsorbed on metal surfaces [1]. In this work we outline how and why similar effects should be achievable in vibrational electron energy-loss spectroscopy (EELS) of nanoparticle adsorbates in the scanning transmission electron microscope (STEM). To this end, we develop a classical model in which the adsorbate is treated as a point dipole on the surface of a metallic dielectric nanoparticle. By applying self-consistent boundary conditions, we find neatly separable expressions for the nanoparticle spectrum and its molecular addition. For physically plausible parameters, molecules adsorbed on the sharp tips of prolate spheroidal nanoparticles yield signal enhancements of hundreds to several thousand times [2].

The sharp spheroidal tip is a special case of the general problem of nanoparticle shape effects. As Lourenço-Martins and Kociak have pointed out [3], there is a close analogy between the surface phonon and surface plasmon modes. Figure 1 illustrates the relationship of nanoparticle geometry, dielectric function, and observed spectra. Solutions to Laplace's equation for a particular geometry dictate the shapes of the harmonic surface modes, along with the values of the dielectric function defining the mode frequencies. Inverting the dielectric function gives us these surface mode frequencies, which all lie between the low-frequency transverse limit and the high-frequency longitudinal limit. Since these modes define surface charges, they also can give us the electric potential acting on the passing probe electron, which translates to various probabilities of energy loss at $\hbar \omega$, where $\omega$ is the surface mode frequency.

In our model for adsorbate signal enhancement (which is based on the long-wavelength theory of Born and Huang [4] in the electrostatic approximation), the molecule is positioned just outside the surface of a Drude nanoparticle. The molecule is driven both by the direct Coulomb field of the beam electron and by the beam-induced field of the nanoparticle. The latter field tends to dominate for aloof geometries such as those presented here. The main enhancement then arises from the nanoparticle's altered field, leading to a molecular signal enhancement roughly proportional to the square of the electric field at the adsorption site. Our model predicts weaker, symmetric line shapes for the spectra of molecules whose resonance frequency is far separated from that of the nanoparticle's surface plasmons, and stronger, Fano-type asymmetric line shapes for molecules whose frequency overlaps the nanoparticle's surface plasmons.

The results displayed in Figure 2, illustrating enhancement effects in the symmetric-lineshape regime, well below surface plasmon energies, are in good agreement with the recent work of Konečná et al. [5], who used the dielectric formalism to describe the STEM-EELS of a thin molecular layer on metallic nanorod tips. We have used parameters for the surface molecule roughly matching those of $\mathrm{H}_{2}$, and Drude parameters for the nanoparticle matching those of silver. When the nanoparticle is tuned to line up certain surface plasmon frequencies with the molecular frequency, this leads to even larger signal enhancements, up to several thousand times. These enhancement effects could be a powerful aide in characterizing such surface-functionalized nanoparticles as are used in chemical sensing. 


\section{References:}

[1] P.G. Etchegoin and E.C. Le Ru, "Basic Electromagnetic Theory of SERS," in Surface Enhanced Raman Spectroscopy (John Wiley \& Sons, Ltd, 2010), Chap. 1, pp. 1-37.

[2] D. Kordahl and C. Dwyer, Phys. Rev. B, in review.

[3] H. Lourenço-Martins and M. Kociak, Phys. Rev. X 7, 041059 (2017).

[4] M. Born and K. Huang, Dynamical Theory of Crystal Lattices (Oxford University Press, 1968).

[5] A. Konečná, T. Neuman, J. Aizpurua, and R. Hillenbrand, ACS Nano 12, 4775 (2018).

Figure 1.

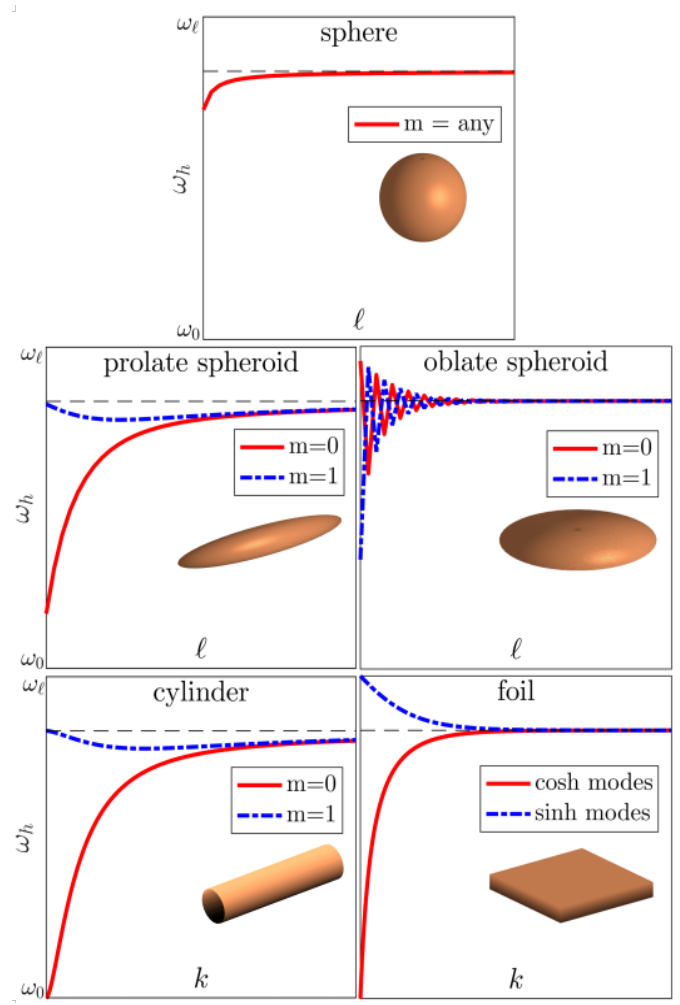

Figure 2.
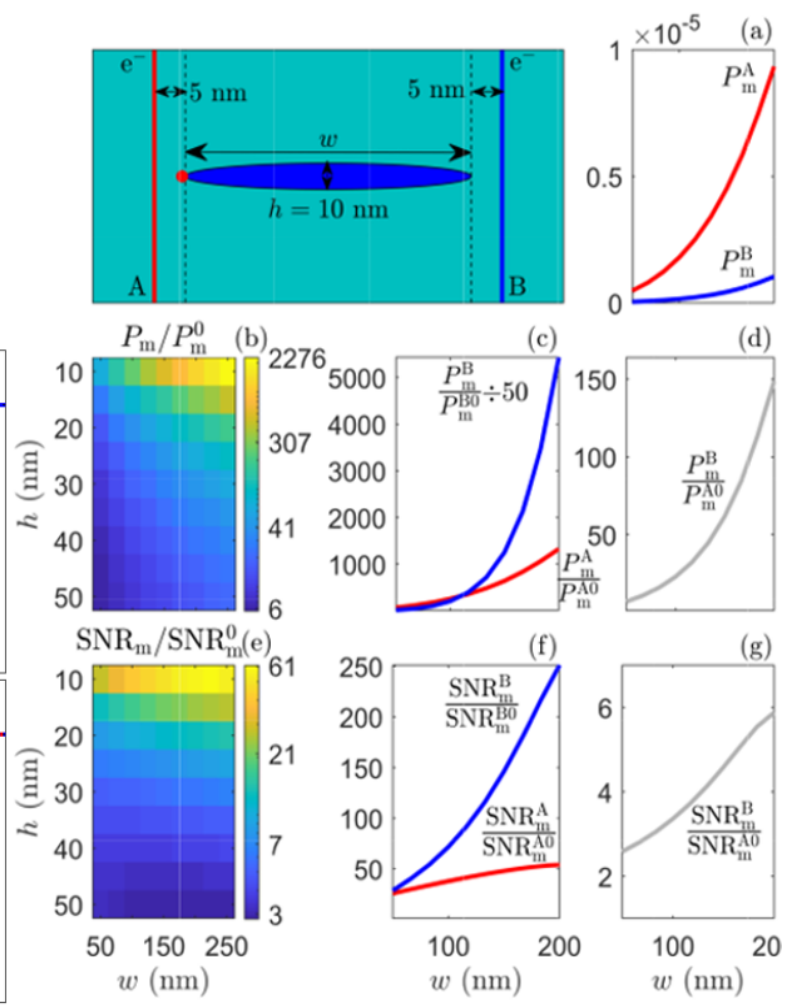

Figure 1. Surface mode frequencies for five nanoparticle shapes. In all cases these frequencies lie between the transverse $\omega_{0}$ and longitudinal $\omega_{1}$ frequencies, and they are associated with the region of the dielectric function with a negative real part.

Figure 2. The red dot represents the adsorbed molecule on the tip of a prolate spheroidal nanoparticle, while A and B refer to "near" and "far" beam positions. (a) EEL probabilities increase with increasing particle length $(w)$, for electron beams positioned at the near and far ends of the nanoparticle. (b) The signal enhancement (defined as the ratio of the molecular signal with the nanoparticle to the molecular signal without the nanoparticle) for beam position A at a variety of thicknesses $(h)$ and lengths $(w)$. The signal without the particle for beam B is expected to be small, since the molecule is far from the beam, so the defined enhancements for beam B are enormous relative to those of beam A (c). Defining the enhancement for beam B to be relative to the bare molecular signal of beam A gives a much more modest result (d). (e-g) The estimated signal-to-noise enhancements (analogous to (b-d)) are also shown. 